\title{
Dispatches from SfN 2021: Mitochondria, Rett therapy and oxytocin
}

\author{
BY ANGIE VOYLES ASKHAM, LAURA DATTARO, ANNA GOSHUA, PETER HESS
}

11 NOVEMBER 2021

The 2021 Society for Neuroscience annual meeting (SfN) wrapped up yesterday, after four packed days of virtual presentations. The in-person portion of the event was called off a month ago because of the ongoing coronavirus pandemic.

A slew of technical glitches plagued the online offerings, but Spectrum's reporters dialed in to a range of autism-related talks, writing up new findings about maternal auto-antibodies, SCN2A mutations and possible gene therapy approaches for Pitt-Hopkins syndrome, among other topics.

The reports below give quick takes on some other autism-related developments of note from the conference.

\section{Mitochondrial stress:}

Researchers are increasingly linking problems with mitochondria to autism and related genetic conditions. For example, previous work has shown that mitochondrial function is disrupted in brain cells missing the gene MECP2, which causes Rett syndrome. In new work presented at SfN this week, the same researchers improved mitochondrial function in mice that model Rett syndrome by injecting the animals with the oral anesthetic dyclonine. The treatment made the mice live longer and improved their breathing, motor skills and limb strength. Boosting the expression of the antioxidant enzyme catalase in the animals yielded similar results.

\section{Mitochondrial ROS-induced stress is a therapeutic target for Rett syndrome}

\section{Genes guiding genes:}


SCN2A, a top autism-linked gene, codes for a protein channel called NaV1.2 that helps neurons propagate electrical signals. The channel's most active location along a neuron, however, changes as the cell matures. A new analysis of gene expression in neurons found that another autism-linked gene, ANK2, helps regulate expression of the channel in the correct places at the appropriate times during development. In particular, ANK2 helps guide NaV1.2 toward neuronal branches called dendrites, and cells with low levels of either SCN2A or ANK2 have mishappen dendrites, the researchers showed.

The ASD-associated genes ANK2 and SCN2A converge to co-regulate dendritic excitability in neocortical pyramidal neurons

\section{RNA therapy:}

The autism-linked genetic conditions Angelman and Prader-Willi syndromes both result from mutations on one copy of chromosome 15. Gene therapies for these conditions have aimed to activate the mutated genes' counterparts on the remaining chromosome, which are typically silenced by a stretch of noncoding RNA. A new screen of small interfering RNAs has revealed thousands of possible snippets that could neutralize the silencing RNA; some of these candidates increase expression of several genes linked to the conditions, including UBE3A, SNORD116 and SNRPN, in human brain cells grown in lab dishes.

\section{RNA interference to treat neurological genetic disorders}

Gene boost: An RNA-based gene therapy increases expression of the UBE3A gene in the cerebellum of mice that model Angelman syndrome.

Courtesy of Brian Butler / UTSW

\section{Rett treatment considerations:}

Mice carrying a mutation in the gene MECP2 show traits associated with Rett syndrome. Boosting MECP2 levels in mice reverses those traits, but too much MECP2 in the brain can also be detrimental - and those effects may differ by sex. A new study in mice highlights one such difference: Genetically supplementing MECP2 levels caused male Rett mice to behave like wildtype mice, but their female counterparts showed signs of altered motor coordination. The findings suggest that increasing MECP2 levels in girls and women with Rett syndrome could have negative effects on motor function, the researchers say. 


\section{Subgroup dynamics:}

Autism is a highly heterogeneous condition that may comprise distinct subgroups with different molecular and genetic roots. To identify some of these subgroups, researchers compared the behavioral traits and resting state brain connectivity patterns of 299 autistic people and 907 neurotypical controls. The autistic participants fell into one of four groups, the team found, based on measurements of their behavioral traits. Each subgroup showed distinct changes in brain connectivity when compared with controls, and that connectivity reflected different patterns of increased expression of autism-related genes - pointing to potential genetic underpinnings of the subtypes.

\section{Defining neurophysiological subgroups in autism spectrum disorder using resting-state connectivity}

\section{Removing repeats:}

Fragile $\mathrm{X}$ syndrome - the most common inherited form of intellectual disability and often accompanied by autism - is caused by excessive repeats of the DNA letters 'CGG' in the FMR1 gene. Most people have 6 to 54 trinucleotide repeats, whereas people with fragile $X$ syndrome have more than 200. In a new analysis, researchers used embryonic stem cells to generate neurons with no CGG repeats in FMR1. Removing the repeats did not affect the expression of FMR1 messenger RNA (mRNA) or the protein it encodes, but it did result in FMR1 mRNA clustering in neuronal projections early in the neurons' growth, suggesting the CGG repeats play a role in early neuronal development.

\section{Function of the trinucleotide (CGG) repeats within the FMR1 gene in human neurons}

\section{Releasing oxytocin:}

Research in prairie voles has suggested that the neuropeptide oxytocin enhances social behavior and bonding, supporting the idea of using it as a therapy for autistic people. But in October, a phase 2 clinical trial showed that chronic intranasal oxytocin did not increase social behaviors in autistic children. To try to stimulate endogenous oxytocin release instead, researchers injected a peptide called melanotan II into the ventricles of prairie voles' brains. The treatment boosted the activity of cells in the prefrontal cortex during social interaction, as measured by levels of the protein c-FOS. Administering the peptide before behavioral therapy could yield greater effects than the drug on its own, the team says. 


\section{Brain games:}

Intranasal doses of oxytocin increased activity in brain areas involved in emotional processing in autistic adult men during a computer game task, a new unpublished study shows. Thirty-two participants, each with an intelligence quotient above 70, played a virtual ball game in which they received feedback from other players and a computer program. Over five sessions, researchers slowly increased the oxytocin dose. Brain scans showed that higher doses were associated with greater activity in the amygdala, ventral anterior insula and nucleus accumbens when the participants received human feedback only. These findings suggest that, despite its mixed results in clinical trials, oxytocin could augment emotional processing in autistic people, the researchers say.

\section{Dose-dependent effects of oxytocin on brain function during an interactive social game in} autism spectrum disorder

\section{Plasticity and protein synthesis:}

Several animal models of autism, including mice with a mutation in the SHANK3 gene, show disruptions in a form of synaptic plasticity called homeostatic scaling, which allows neurons to maintain a steady firing rate despite shifts in their overall excitability. A new unpublished study shows that SHANK3 mice also have lowered protein synthesis compared with wildtype mice. The researchers observed alterations in the mTOR pathway, which regulates protein synthesis and may mediate homeostatic scaling in autism. The results suggest that deficits in the mTOR network may account for the altered synaptic plasticity seen in some animal models of autism.

The mTOR protein interaction network responds to homeostatic scaling and is disrupted in SHANK3 knockout mice

\section{Altering GABA activity:}

Some people with autism or related conditions show atypical levels of gamma-aminobutyric acid (GABA), an inhibitory neurotransmitter, or of GABA receptors - changes that contribute to a brain signaling imbalance. To correct such GABA-related imbalances, researchers at the Swiss drug company Roche used experimental drugs to activate or inhibit one type of GABA receptor, called GABA-A, in several mouse models of autism or related conditions. A molecule that blocks the receptor's activity normalized social behaviors and flexibility in spatial learning in a mouse model of dup15q syndrome; it also decreased unusually strong brain waves called beta oscillations, which are a hallmark of the syndrome. By contrast, a drug that increases GABA-A activity normalized repetitive behaviors in two different mouse models: an asocial inbred strain called BTBR and mice missing CNTNAP2, a gene linked to autism. 


\section{Spectrum | Autism Research News}

https://www.spectrumnews.org

Therapeutic potential of GABAA alpha5 subunit-containing receptors in neurodevelopmental disorders

\section{Neuronal cell marker:}

Cell models of autism are useful for understanding how the condition arises and for developing individualized therapies. Researchers performed RNA sequencing on 168,032 neural progenitor cells derived from three autistic and three non-autistic children, revealing 14 clusters of cell types based on gene expression patterns. Among the cells derived from the autistic children, a noncoding snippet of RNA called miR-128 that regulates gene expression was downregulated and may be a potential therapeutic target, the researchers say.

\section{Characterizing human iPSC-derived neural progenitor cells in idiopathic autism}

\section{Cautionary mouse tales:}

Two sets of unpublished findings presented at SfN provide stark reminders of how subtle factors can skew the results in mouse studies: Among mice with two mutated copies of the autism-linked gene CAMK2A, the bedding they are raised on - coarse corncob or soft paper - affects their touch sensitivity and texture preference in adulthood, according to one study. And background lineage sways physical characteristics and autism-related phenotypes in mice missing a copy of the autismlinked gene CHD8, according to the other. This variability could help explain why people with CHD8 mutations can express traits so differently.

\section{Impact of bedding material on tactile behavioral phenotypes of a CAMK2A E183V mutant} mouse model of ASD

Modeling phenotypic heterogeneity in autism spectrum disorder by leveraging a mouse model of global genetic diversity, the Collaborative Cross Genetic Reference Panel, with CHD8 haploinsufficiency

\section{Exhibitor furor:}

A group of exhibitors who signed up to promote their businesses at the canceled in-person portion of the conference are calling on SfN to refund their booth fees, citing poor traffic and technical issues with 'virtual booths.' When the meeting was transitioned to an all-virtual event, SfN offered exhibitors the option to display a virtual booth for a 50 percent refund of their original exhibit space fee, which ranged from $\$ 5,430$ to $\$ 6,510$ for commercial exhibitors, or apply the fee toward advertising space. There was no option to pull out for a full refund as there was for conference attendees. Frustrated exhibitors have published an open letter calling on SfN to refund exhibitors 100 percent of their fees. 


\section{Spectrum | Autism Research News}

https://www.spectrumnews.org

Neuroscience 2021 Exhibitor Full Refund

Read more reports from the 2021 Society for Neuroscience virtual annual meeting.

Cite this article: https://doi.org/10.53053/RNDH5082 